\title{
Isolation of High Density Lipoproteins from Rat Intestinal Epithelial Cells
}

\author{
Arthur M. Magun, Thomas A. Brasitus, and Robert M. Glickman
}

Gastrointestinal Division, Department of Medicine, Columbia University College of Physicians and Surgeons, New York 10032

\begin{abstract}
Previous studies have defined forms of high density lipoproteins (HDL) in rat mesenteric lymph, suggesting that they have a secretory origin. This study describes the isolation and characterization of intestinal intracellular HDL.

Two preparations were made as follows. (a) Rat enterocytes were isolated and a Golgi organelle fraction was prepared. (b) Cell homogenates were subjected to nitrogen cavitation and a cytoplasmic fraction was prepared. Lipoproteins were isolated from both preparations by sequential ultracentrifugation. When the HDL fraction $(1.07-1.21 \mathrm{~g} / \mathrm{ml})$ was subjected to isopyknic density gradient ultracentrifugation, a peak of apoproteins A-I and B (apoA-I and apoB, respectively) was found at a density of $1.11-1.14 \mathrm{~g} / \mathrm{ml}$. Electron microscopy of the fraction showed spherical particles ranging in size from 6 to $13 \mathrm{~nm}$. Immunoelectrophoresis revealed a precipitin arc in the alpha region against apoA-I which extended into the pre-beta region where a precipitin arc against apoB was also seen. ApoB antisera depleted the pre-beta particles whereas the alpha migrating particles remained. Lipid analysis of the whole HDL fraction revealed phospholipid, cholesteryl ester, and triglyceride as the major lipids. $\left[{ }^{3} \mathrm{H}\right]$ leucine was then administered into the duodenum and a radiolabeled intracellular HDL fraction was isolated. The newly synthesized apoproteins of the HDL fraction, as determined by gel electrophoresis, were apoB, apoA-I, and apolipoprotein A-IV (apoA-IV). Immunoprecipitation of the apoB particles revealed apoA-I and apoA-IV in the supernatant.

These data demonstrate that there are at least two intracellular intestinal forms of HDL particles, one of which contains apoB. The other particle contains apoA-I and apoAIV, has alpha mobility, is spherical, and resembles a particle found in the lymph.
\end{abstract}

\section{Introduction}

Identification of the apoproteins synthesized by the intestine and characterization of rat intestinal mesenteric lymph lipoproteins have suggested that the intestine is a site of origin of high density lipoproteins (HDL). Windmueller and Wu have

This work was presented in part at the Annual Meeting of the American Society for Clinical Investigation, 1 May 1983, and published in abstract (1983. Clin. Res. 31:503A).

Dr. Brasitus is currently with the Division of Gastroenterology, Michael Reese Hospital, Chicago, IL.

Received for publication $28 \mathrm{July} 1983$ and in revised form $18 \mathrm{July}$ 1984.

J. Clin. Invest.

(c) The American Society for Clinical Investigation, Inc. $0021-9738 / 85 / 01 / 0209 / 10 \quad \$ 1.00$

Volume 75, January 1985, 209-218 shown that $50 \%$ of rat plasma apolipoproteinA-I (apoA-I), ${ }^{1}$ the principal apoprotein of $\mathrm{HDL}$, is synthesized by the intestine (1). Studies from this laboratory have shown that during fasting, $50-85 \%$ of apoA-I was in the $d>1.006 \mathrm{~g} / \mathrm{ml}$ fraction of lymph and that $75 \%$ of the apoA-I of lymph HDL was synthesized by the intestine, suggesting that newly synthesized apoA-I might be secreted on an $\mathrm{HDL}$ particle $(2,3)$. Three forms of HDL particles have been isolated from the lympha 10-12-nm spherical particle resembling plasma HDL, a discoidal particle, and a small spherical particle. The 10-12$\mathrm{nm}$ spherical particle is the predominant lymph HDL and has a density of $1.095 \mathrm{~g} / \mathrm{ml}$ (4). The discoidal particle is rich in phospholipid, contains apoA-I as its major apoprotein, and structurally resembles discoidal particles seen in the effluent of hepatic perfusates (5). Forester et al. characterized a small (7.8 $\mathrm{nm}$ ) spherical HDL particle in lymph which had a density of $1.13-1.18 \mathrm{~g} / \mathrm{ml}$, was rich in apoA-I and phospholipid, and had a cholesteryl ester core (4). The cholesteryl ester core of the particle incorporated duodenally infused ${ }^{3} \mathrm{H}$-cholesterol, suggesting that the particle was newly synthesized in the intestine.

Thus, although lymph HDL particles appear to be synthesized and secreted by the intestine, alternative explanations are possible. For example, HDL particles might be formed through the interaction of newly secreted chylomicron surface components with lipoproteins in lymph that derive from plasma. Isolation of HDL particles from enterocytes would clarify this question, particularly since chylomicrons and very low density lipoprotein (VLDL)-sized particles, but not HDL particles, have been directly isolated from within intestinal epithelial cells (6-8).

The present study was undertaken to examine the hypothesis that if the intestine secretes HDL, there should be a form of HDL in the epithelial cell that resembles particles found in the lymph. We describe in this paper methodology to isolate lipoproteins from within intestinal cells which was used to isolate and characterize nascent forms of intracellular HDL. We have found that there is a newly synthesized HDL particle in the cell which resembles the small spherical HDL particle found in the lymph. In addition, there is another intracellular particle isolated in the HDL density range which contains apoB as the predominant apoprotein.

\section{Methods}

\section{HDL isolation procedures}

Isolation of epithelial cells. The method to isolate epithelial cells from the rat intestine is a modification of the Weiser technique (9). Male rats (200-250 g) of the Sprague-Dawley strain were used in all experiments. The rats were housed in polyethylene cages and allowed rat

1. Abbreviations used in this paper: apo A-I, apo A-IV, and apo B-I, apolipoproteins A-I, A-IV, and B-I, respectively; DTT, dithiothreitol; LCAT, lecithin/cholesterol acyl transferase; $R_{f}$, retardation factor; TLC, thin-layer chromatography. 
chow and water ad lib. All experiments began between 8 and 10 a.m. Each rat was killed by a stun blow to the head followed by cervical dislocation. Immediately thereafter, the peritoneal cavity was opened and the whole small intestine was removed. The intestine was rinsed free of chyme with a solution of $150 \mathrm{mM} \mathrm{NaCl}$ and $1 \mathrm{mM}$ dithiothreitol (DTT). One end of the intestine was then tied and filled with a citrate solution $(27 \mathrm{mM}$ sodium citrate, $96 \mathrm{mM}$ sodium chloride, $1.5 \mathrm{mM}$ potassium chloride, $8 \mathrm{mM}$ potassium dihydrogen phosphate, $5.6 \mathrm{mM}$ sodium phosphate dibasic, pH 7.2). The fluid-filled intestinal loop was placed into a beaker filled with the same citrate buffer and shaken in a water bath $(60$ oscillations $/ \mathrm{min})$ at $37^{\circ} \mathrm{C}$ for $15 \mathrm{~min}$. The intestine was then removed from the beaker, emptied, and filled with a phosphate-buffered saline (PBS)-EDTA-DTT solution (145 mM NaCl, $4 \mathrm{mM} \mathrm{KCl}, 5 \mathrm{mM} \mathrm{NaH} \mathrm{PO}_{4}, 5 \mathrm{mM} \mathrm{Na} 2 \mathrm{HPO}_{4}, 1.5 \mathrm{mM}$ EDTA, 0.5 mM DTT). The loop was replaced into the citrate buffer in the water bath and incubated at $37^{\circ} \mathrm{C}$ for $30 \mathrm{~min}$. After the incubation, the intestine was removed, one end was unclamped, and the contents of the loop were emptied into a beaker by passage of the intestine once over the lip of the beaker while mild pressure against the serosal surface was maintained. Cells were pelleted at $1,000 \mathrm{~g}$ for $5 \mathrm{~min}$ in a SS34 Sorvall rotor in a Sorvall centrifuge (DuPont Instruments-Sorvall Biomedical Div., Newtown, CT), resuspended in cold isotonic saline, and then pelleted again at $1,000 \mathrm{~g}$ for $5 \mathrm{~min}$ to wash the cells free of any lymph or serum protein. The wash was repeated twice.

Isolation of intracellular lipoproteins from a Golgi preparation. This procedure is based on a method to isolate and rupture Golgi membranes as described by Howell and Palade $(10,11) .13-15$ rats were used for each Golgi preparation. Epithelial cells were isolated, washed, and pelleted as above. The cell pellet was suspended in $400 \mathrm{ml}$ of cold isotonic sucrose $(0.25 \mathrm{M})$ and the cells were dispersed by vortex. The suspension was placed in a nitrogen cavitation apparatus (Parr Instrument Co., Moline, IL) at 800 psi for 15 min to rupture the cells, after which the membrane fragments were pelleted at $10,000 \mathrm{~g}$ in a SS34 Sorvall rotor for $10 \mathrm{~min}$. The supernatant was centrifuged at 100,000 $\boldsymbol{g}$ for $1 \mathrm{~h}$, forming a microsomal pellet. The pellet was resuspended in isotonic sucrose, and $2.0 \mathrm{M}$ sucrose was added to reach a density equivalent to that of $1.22 \mathrm{M}$ sucrose, as measured by the refractive index (1.3920). A density gradient was then prepared by overlaying 9 $\mathrm{ml}$ of the microsomal $1.22 \mathrm{M}$ fraction with 9-ml aliquots of 1.15 , 0.86 , and $0.25 \mathrm{M}$ sucrose solutions. The gradient was centrifuged in an SW27 rotor (Beckman Instruments, CA) at 25,000 rpm for $3 \mathrm{~h}$. The band that formed at the interface of the $0.25 / 0.86 \mathrm{M}$ solutions was the putative Golgi fraction. It showed a 20-25-fold step-up in galactosyl transferase activity over that of the microsomal fraction. This band is thought to contain the lighter elements of the Golgi fraction (10). The density of the fraction was readjusted to that of isotonic sucrose $(0.25 \mathrm{M})$ by the addition of ice-cold water until a refractive index of 1.3450 was reached, allowing the Golgi organelles to expand. The organelles were then pelleted at $100,000 \mathrm{~g}$ for $1 \mathrm{~h}$ and the pellet was suspended in $12 \mathrm{ml}$ of $100 \mathrm{mM}$ ice-cold sodium carbonate, $\mathrm{pH}$ 11.3. The organelles were dispersed in the buffer with 10 strokes in a tight glass Dounce homogenizer $(40 \mathrm{ml}$; Wheaton Scientific, Millville, NJ) and kept on ice for $45 \mathrm{~min}$, and then the ruptured organelle fragments were pelleted at $100,000 \mathrm{~g}$ for $1 \mathrm{~h}$. Golgi organelle lipoproteins were isolated from the supernatant.

Isolation of intracellular lipoproteins from a cytoplasmic preparation. A greater yield of intracellular lipoproteins could be obtained by an isolation procedure that harvested these lipoproteins from a total cellular cytoplasmic homogenate. Each cytoplasmic preparation used 8-12 rats. After isolation and washing of the epithelial cells, the cell pellet was suspended in $250-300 \mathrm{ml}$ of cold hypotonic veronal buffer (pH 8.3, $37.5 \mathrm{mM} \mathrm{Na}$ diethylbarbiturate and $7.3 \mathrm{mM}$ barbituric acid). The cells were kept at $5-10^{\circ} \mathrm{C}$ at all times during the isolation procedure. The cells were dispersed in the solution by five strokes in a glass Dounce homogenizer. The solution was then placed into a cold nitrogen cavitation apparatus at 2,000 psi for $15 \mathrm{~min}$. After the cells were passed through the cavitation apparatus, 40-ml aliquots were sonicated for $15 \mathrm{~s}$ on ice in a Bronson-type sonicator, with a microtip, at 3.5 intensity and $50 \%$ setting. The solution was then passed again through the nitrogen cavitation apparatus for $15 \mathrm{~min}$ at $2,000 \mathrm{psi}$ and then centrifuged at $100,000 \mathrm{~g}$ for $1 \mathrm{~h}$ in a $50.2 \mathrm{Ti}$ rotor in a Beckman ultracentrifuge (Beckman Instruments). The supernatant of this cytoplasmic fraction contained lipoproteins liberated from intracellular organelles and vesicles.

Isolation of an HDL fraction from the Golgi or cytoplasmic preparation. Lipoproteins liberated from intracellular organelles from the Golgi or cytoplasmic preparation were isolated by differential ultracentrifugation. The density of the final supernatant from either preparation was raised to $1.070 \mathrm{~g} / \mathrm{ml}$ by the addition of $\mathrm{NaBr}$. The solution was centrifuged for $16 \mathrm{~h}$ in an SW27 rotor at $25,000 \mathrm{rpm}$ $\left(8.2 \times 10^{7} \mathrm{~g} \mathrm{~min}\right)$. For the Golgi preparations, an SW40 rotor was used. Use of a swinging bucket rotor at this step allows membrane bound proteins to be pelleted and thereby separated from liberated lipoproteins. The 1.070 top, which contains chylomicrons, VLDL, and low density lipoprotein, was removed by pipette, and the 1.070 infranatant was raised to a density of $1.21 \mathrm{~g} / \mathrm{ml}$ by the addition of $\mathrm{NaBr}$. The solution was centrifuged for $44-48 \mathrm{~h}$ in a $50.2 \mathrm{Ti}$ rotor at $44,000 \mathrm{rpm}\left(4.5 \times 10^{8} \mathrm{~g} \mathrm{~min}\right)$. The 1.21 top was removed by pipette and concentrated to a volume of $2-4 \mathrm{ml}$ by vacuum dialysis in a collodion bag apparatus (Schleicher \& Schuell, Inc., Keene, NH). This fraction is hereafter referred to as the concentrated whole (1.07-1.21) HDL fraction. This fraction was subfractionated by isopyknic density gradient ultracentrifugation as described (12). A 1.3-ml aliquot of the concentrated whole HDL fraction was raised to a density of $1.20 \mathrm{~g} / \mathrm{ml}$ with $\mathrm{NaBr}$ (as the density decreases during vacuum dialysis) and overlayed with 1-ml aliquots of 1.16-, 1.13-, 1.10-, and 1.08-g/ml NaBr solutions and centrifuged for $72 \mathrm{~h}$ in an SW50.1 rotor (Beckman Instruments) at $49,000 \mathrm{rpm}$. Fractions of $300-400 \mu \mathrm{l}$ were removed by pipette from the density gradient for analysis. The densities of each fraction were determined by refractometry and then dialyzed into water containing $0.04 \%$ EDTA, $0.02 \%$ sodium azide, and adjusted to a pH of 8.3 with $\mathrm{NH}_{4} \mathrm{OH}$.

Isolation of HDL from bile-ligated animals. Two Golgi (15 rats) and two cytoplasmic preparations (11 and 12 rats) were made from bile-ligated animals. A laparotomy was performed on each rat by the use of ether-pentobarbitol anesthesia, and the common bile duct was ligated. An intraperitoneal dose of $250 \mu \mathrm{g}$ of gentamicin was administered. The peritoneum was closed with surgical ligatures and the animals were allowed to recover in their cages with food and water ad lib. over the next $48 \mathrm{~h}$. When the peritoneal cavity was reopened after the animals were killed by cervical dislocation, the continued presence of the ligature around the common duct was verified to ensure that all animals used had indeed been bile ligated. Each intestine was removed and a lipoprotein fraction was isolated by the cytoplasmic preparation method.

Isolation of an HDL fraction from lymph chylomicrons. This experiment was designed to determine whether intracellular HDL particles could be formed from intracellular chylomicrons during the cytoplasmic isolation procedure. A cytoplasmic preparation using eight rats was performed. The intracellular lipoproteins were isolated by sequential ultracentrifugation and removed by pipette, as described above. The $1.21-\mathrm{g} / \mathrm{ml}$ bottom of this preparation, subsequently dialyzed, contained all cytoplasmic enzymes and proteins except for lipoproteins. Main mesenteric rat lymph was collected overnight on ice as previously described (13). The lymph was defibrinated and centrifuged for $16 \mathrm{~h}$ in an SW27 rotor at $25,000 \mathrm{rpm}$ to isolate chylomicrons and VLDL. $12 \mathrm{ml}$ of this lymph chylomicron-VLDL fraction was then added to the cytoplasmic $1.21-\mathrm{g} / \mathrm{ml}$ lipoprotein-free bottom. The solution was processed as if it were a newly isolated suspension of epithelial cells in buffer; i.e., it was passed through the cavitation apparatus, sonicated, and passed through the cavitation apparatus again. Sequential ultracentrifugation was then performed on the final supernatant of this cytoplasmic preparation to isolate any particles with a density of 1.07$1.21 \mathrm{~g} / \mathrm{ml}$. As the starting solution contained no lipoproteins other 
than chylomicrons and VLDL, any particles found in the HDL range would presumably have been produced from these triglyceride-rich lipoproteins.

$\left[{ }^{3} \mathrm{H}\right.$ ]leucine incorporation into $H D L$. Five rats were fasted overnight. They were then anesthetized, and a laparotomy was performed. A ligature was placed around the midjejunum and $4 \mathrm{mCi}$ of $\left[{ }^{3} \mathrm{H}\right] l e u c i n e$ $\left(4,5\left[{ }^{3} \mathrm{H}\right]\right.$ leucine, $137 \mathrm{Ci} / \mathrm{mmol}$; Amersham Corp., Arlington Heights, IL) in a volume of $4 \mathrm{ml}$ was injected into the duodenum at the level of the ligament of Treitz, creating a $\left[{ }^{3} \mathrm{H}\right]$ leucine-filled loop of distal duodenum and jejunum. The bowel was not distended. The abdomen was stapled closed and after 9 min the animals were killed by cervical dislocation. The intestinal loop was removed and unabsorbed $\left[{ }^{3} \mathrm{H}\right]$ leucine was collected by the opening of one end of the loop and draining of the contents. The intestine was then filled with citrate solution and the radiolabeled intestinal cells were isolated as described above for unlabeled cells. The isolated cells were washed and suspended in $100 \mathrm{ml}$ of veronal buffer. A cytoplasmic preparation was made, and an HDL fraction was isolated, concentrated in a collodion bag, and then. centrifuged on an isopyknic density gradient.

\section{Lipoprotein characterization}

Immunoelectrophoresis. The HDL fraction was dialyzed at $4^{\circ} \mathrm{C}$ against water overnight. Agarose gel immunoelectrophoresis of a $10-\mu l$ aliquot of the sample was performed against antisera to apoA-I and apolipoprotein B (apoB) by standard methods. Each antiserum was produced by isolation of the apoprotein on a polyacrylamide gel and immunization of a rabbit with the gel slice containing the apoprotein mixed with Freund's adjuvant as described (14). The antisera have been previously characterized by this laboratory $(2,14)$.

Immunoprecipitation. To remove apoB-containing particles from the HDL fraction, enough apoB antiserum to remove all apoB was added to the HDL fraction and incubated at $4^{\circ} \mathrm{C}$ for $36-48 \mathrm{~h}$. The solution was then centrifuged in a microcentrifuge (Beckman microcentrifuge 12; Beckman Instruments) at 3,000 rpm for $30 \mathrm{~min}$ and the supernatant was taken for immunoelectrophoretic analysis. Alternatively, apoB containing particles were immunoprecipitated by use of goat anti-rabbit IgG bound to an acrylamide bead as a second antibody (Immunobeads; Bio-Rad Laboratories, Rockville Center, NY). The immunobeads were added $36-48 \mathrm{~h}$ after the first antibody had been added, and the suspension was incubated at $37^{\circ} \mathrm{C}$ for $2 \mathrm{~h}$. The immunoprecipitate was pelleted at $3,000 \mathrm{rpm}$ in a microcentrifuge for $10 \mathrm{~min}$.

Electron microscopy. The Golgi band from the sucrose density gradient was pelleted and suspended in a final concentration of $2 \%$ osmium tetraoxide for $2 \mathrm{~h}$. The stained vesicles were then pelleted, dehydrated, and embedded in Spurr. Silver gold sections were taken and stained with uranyl acetate and lead citrate. The sections were viewed on a JEM-100 CX electron microscope (JCOI, Ltd., Tokyo, Japan).

HDL fractions were negatively stained with $2 \%$ sodium phosphotungstic acid on Formvar-coated copper grids as described (4). Particles were sized directly from random areas of the negative of the micrograph by use of a magnifying eye piece with a reticle.

Polyacrylamide gel electrophoresis. Isolated HDL fractions were delipidated by ethanol-ether $(3 / 2, \mathrm{vol} / \mathrm{vol})$ at $4^{\circ} \mathrm{C}$ and then subjected to sodium dodecyl sulfate (SDS)-polyacrylamide gel electrophoresis according to the method of Fairbanks et al. (15). Isotope incorporation into apoprotein bands was determined by counting of gel slices. The gels were immediately sliced with a gel slicer into $1.5-\mathrm{mm}$ slices and incubated overnight in liquid scintillation solution (3\% Protosol-97\% Econofluor; New England Nuclear, Boston, MA), and radioactivity was determined in a scintillation counter (LS-350; Beckman Instruments).

Apoprotein mass determination by radioimmunoassay (RIA). Total protein was measured by the method of Lowry (16). ApoA-I and apoB were measured by RIA. For the apoA-I RIA, $10 \mu \mathrm{g}$ of apoA-I was iodinated by the chloramine $\mathrm{T}$ method using $1 \mathrm{mCi}$ of ${ }^{125} \mathrm{I}(>350$
$\mathrm{mCi} / \mathrm{ml}$; New England Nuclear) as described previously (3). The RIA was performed by the addition of $100 \mu$ l of sample to $20 \mu \mathrm{l}$ of a $5 \%$ Triton-PBS solution. After a 30 -min incubation at $37^{\circ} \mathrm{C}, 300 \mu \mathrm{l}$ of a solution containing a 1:500 dilution of nonimmune rabbit serum, and $20,000-30,000 \mathrm{cpm}$ of ${ }^{125} \mathrm{I}$-apoA-I was added, after which $100 \mu \mathrm{l}$ of antisera to apoA-I was added. After a $48-\mathrm{h}$ incubation at $4^{\circ} \mathrm{C}$, goat anti-rabbit IgG was added. The immune complex was harvested $16 \mathrm{~h}$ later by centrifugation at $3,000 \mathrm{rpm}$ in a centrifuge (DuPont InstrumentsSorvall Biomedical Div.), resuspended in PBS, and recentrifuged. Radioactivity was measured in a gamma counter (LKB-1270; LKB Instruments, Inc., Gaithersburg, MD). All samples were assayed in triplicate. The working range of the assay was 1-500 ng of apoA-I. The within-assay coefficient of variation was $3.2 \%$, and the betweenassay coefficient of variation was $1.2 \%$. The apoB RIA was similarly performed, with the following modifications: The iodination procedure used $20 \mu \mathrm{g}$ of apoB and was stopped after $10 \mathrm{~s}$ with sodium metabisulfite. A second column of Sephadex G-200 was used in addition to the G25 column to purify the iodinated protein. Silicon-treated tubes were used to minimize nonspecific binding of the iodinated apoB. About $7,000 \mathrm{cpm}$ were added to each tube in the assay. The working range of the assay was $15-3,500 \mathrm{ng}$ of apoB. The within-assay coefficient of variation was $5 \%$, and the between-assay coefficient of variation was $10 \%$.

Lipid analysis. The HDL fraction from an isopyknic density gradient of normal and bile-ligated rats was analyzed for total lipid content by extraction in a chloroform/methanol $(2 / 1, \mathrm{vol} / \mathrm{vol})$ solution according to the method of Folch et al. (17). Lipid composition was determined by quantitative thin-layer chromatography (TLC) as described by Downing and modified by Katz (18). The apolar solvent system was a hexane/ether/acetic acid solution (70:30:1, vol/vol) and the polar solvent was chloroform/methanol/water/acetic acid (65:25:4:1, vol/vol).

The HDL fractions from density gradients of three cytoplasmic preparations were also analyzed for total lipid content. The HDL fraction of each preparation was divided into equal volumes, and lipidfree apoB antiserum was added to one of the aliquots to immunoprecipitate apoB-containing particles. The lipid-free antiserum was prepared by raising the density of the rabbit antiserum to $1.21 \mathrm{~g} / \mathrm{ml}$ and centrifuging it for $72 \mathrm{~h}$ in a SW50.1 rotor. The $1.21 \mathrm{-g} / \mathrm{ml}$ infranatant was then dialyzed into normal saline. An aliquot of the lipid-free antisera revealed only fatty acid by TLC. Immunoprecipitation of the apoB particles was facilitated by use of a second antibody as described above. The second antibody, i.e., the immunobeads, contained lipid, but the supernatant of a control solution containing the immunobeads alone was, after centrifugation of the immunobeads, lipid free, suggesting that the lipid intrinsic to the goat anti-rabbit antisera was adherent to the immunobead. The whole HDL fraction and the supernatant after the immunoprecipitation were extracted as above.

\section{Results}

Isolation of intracellular HDL particles. Fig. $1 A$ is an electron micrograph of the Golgi band $(0.25 / 0.86 \mathrm{M}$ sucrose interface) from the sucrose density gradient. Vesicles are seen filled with lipid. The particles in the vesicles measure from 39 to 274 nm. Particles were liberated from the vesicles and an HDL fraction was prepared. Fig. $1 B$ is an electron micrograph of the concentrated HDL fraction liberated from ruptured Golgi vesicles. Spherical particles of average size $8.2 \mathrm{~nm}$ are seen. There are no discoidal particles or membrane fragments.

HDL subclasses were identified by centrifugation of the concentrated HDL fraction $(1.07-1.21 \mathrm{~g} / \mathrm{ml})$ on isopykinic density gradients. Equal fractions (300-400 $\mu$ l) were removed by pipette from the gradient and assayed for apoA-I and apoB by RIA (Fig. 2). A peak of apoprotein content is seen corre- 

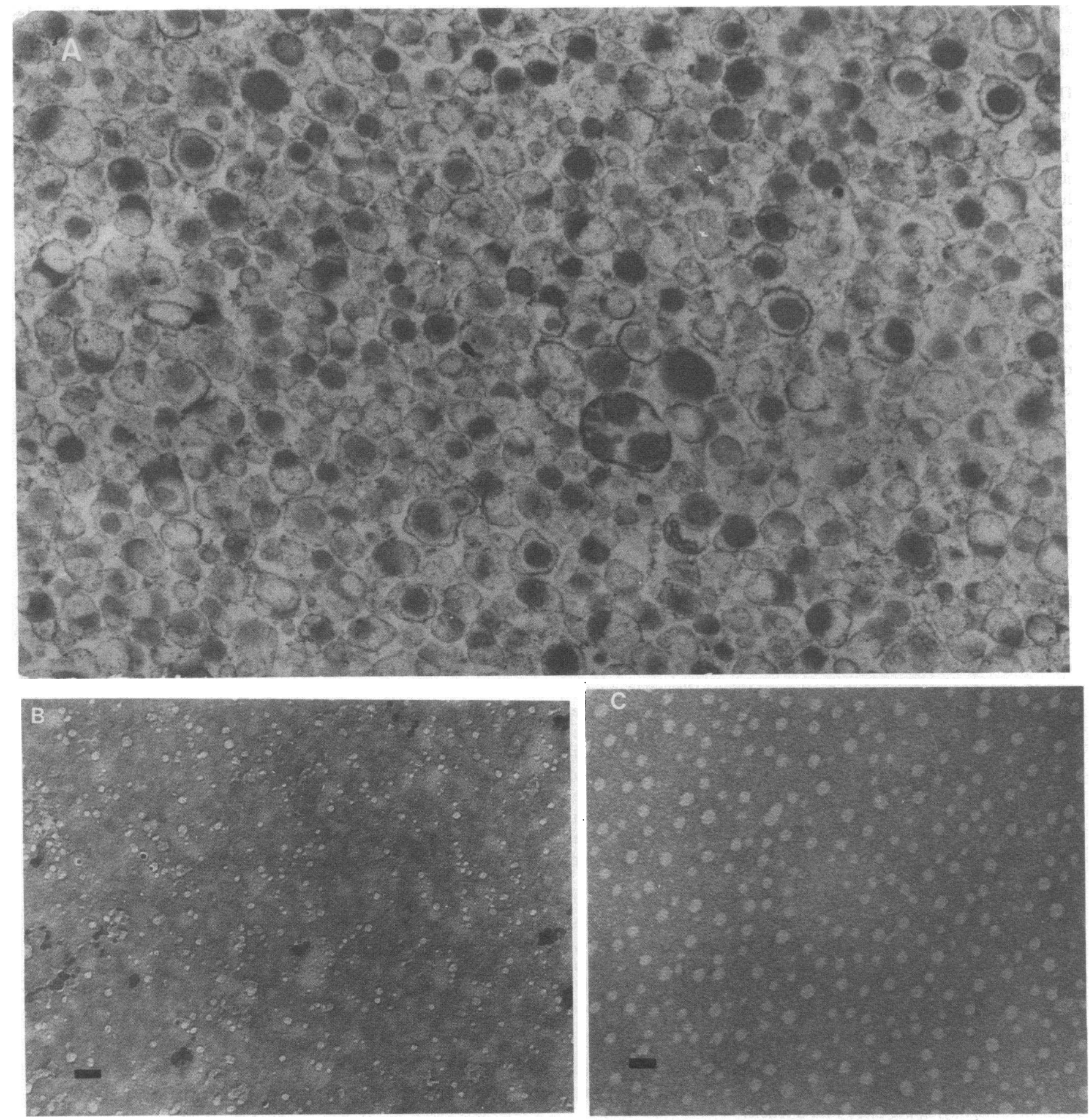

Figure 1. Electron micrographs of Golgi organelles and intracellular HDL from rat enterocytes. $(A)$ The Golgi fraction- $0.25 / 0.86$ interface from the sucrose density gradient-containing vesicles filled with lipid (magnification of 38,000). (B) HDL particles liberated from the

sponding to a density of $1.11-1.14 \mathrm{~g} / \mathrm{ml}$ for both apoproteins. The peak at the top of the gradient represents non-HDL particles $(d<1.07 \mathrm{~g} / \mathrm{ml})$, which presumably were not removed when the 1.070 top was pipetted off after a 16-h centrifugation. The peak at the bottom of the gradient may represent particles of very high density. Alternatively, the peak at the bottom could be free apoprotein that may have been within Golgi organelles or released during centrifugation. (apoA-I, but not
Golgi vesicles with an average size of $8.2 \mathrm{~nm}$. (C) HDL particles from the $1.13 \mathrm{~g} / \mathrm{ml}$ density fraction of a cytoplasmic preparation with average size $11.5 \mathrm{~nm}$. Sizing bar, $25 \mathrm{~nm}$.

apoB, is known to be released during centrifugation.) The apoprotein peak in the middle of the gradient suggests that there is a population of intracellular particles with a density of $1.11-1.14 \mathrm{~g} / \mathrm{ml}$.

The concentrated HDL fraction from one representative Golgi preparation made from 13 rats contained $2.24 \mu \mathrm{g}$ of apoA-I and $11.5 \mu \mathrm{g}$ of apoB. The small protein yields of the Golgi method precluded more than one analysis per prepara- 


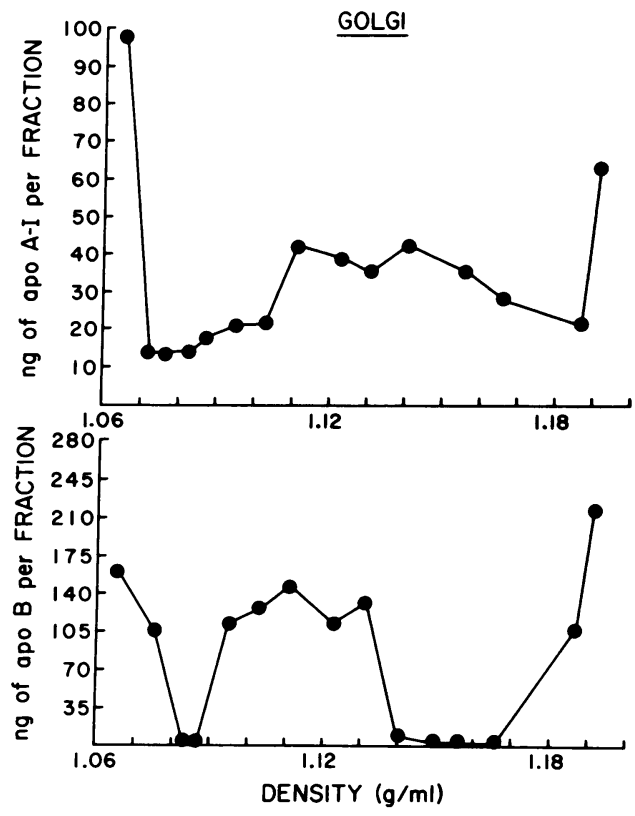

Figure 2. Apoprotein distribution of Golgi HDL particles. The concentrated whole HDL fraction $(1.07-1.21 \mathrm{~g} / \mathrm{ml})$ from a Golgi preparation was loaded onto an isopyknic density gradient, and after a 72 $h$ centrifugation, apoA-I and apoB were measured by RIA across the gradient. The peak in the gradient between densities 1.11 and $1.14 \mathrm{~g} /$ $\mathrm{ml}$ suggests a population of HDL particles. The peak at density 1.07 $\mathrm{g} / \mathrm{ml}$ represents non-HDL particles, and the peak at density $1.19 \mathrm{~g} /$ $\mathrm{ml}$ represents free apoprotein or very high density lipoproteins.

tion. To perform further analyses on intracellular particles, the cytoplasmic method was devised to isolate all intracellular lipoproteins, not just lipoproteins from Golgi vesicles. The concentrated HDL fraction from the cytoplasmic preparations contained from 15-40 $\mu \mathrm{g}$ of apoA-I and 75-200 $\mu \mathrm{g}$ of apoB, i.e., more than 10 times the Golgi yield.

The HDL isolated by the cytoplasmic method were characterized in the same manner as the HDLs from Golgi. The apoprotein distribution across a density gradient from a cytoplasmic preparation is seen in Fig. 3. There is a peak of apoA$I$ and of apoB at $1.12 \mathrm{~g} / \mathrm{ml}$. The peak fraction examined by negative stain electron microscopy revealed spherical particles with an average size of $11.5 \mathrm{~nm}$ (Fig. 1C). About $15 \%$ of the particles were 7-8 $\mathrm{nm}$. The cytoplasmic preparation then, yielded an HDL fraction that was slightly larger (11.5 vs. 8.2 $\mathrm{nm}$ ) and somewhat less dense (1.12 vs. $1.11-1.14 \mathrm{~g} / \mathrm{ml})$ than HDL from Golgi.

Isolation of HDL from bile-ligated animals and lymph chylomicrons. The possibility that isolated HDL particles were artifactual fragments of chylomicrons produced during the procedure was examined by two different approaches. The first approach was to isolate HDL from bile-ligated animals. Bile diversion depletes intestinal cells of triglyceride-rich lipoproteins and reduces lymph triglyceride output by $80 \%$, markedly diminishing production of lymph chylomicrons. The apoprotein distribution of apoA-I and apoB across a density gradient of bile-ligated animals from a cytoplasmic preparation is seen in Fig. 4. The peak density $(1.13 \mathrm{~g} / \mathrm{ml})$ of HDL from bile-ligated animals is similar to that of normal rats, suggesting that marked reduction of intracellular lipid does not significantly alter production of an intestinal HDL particle.

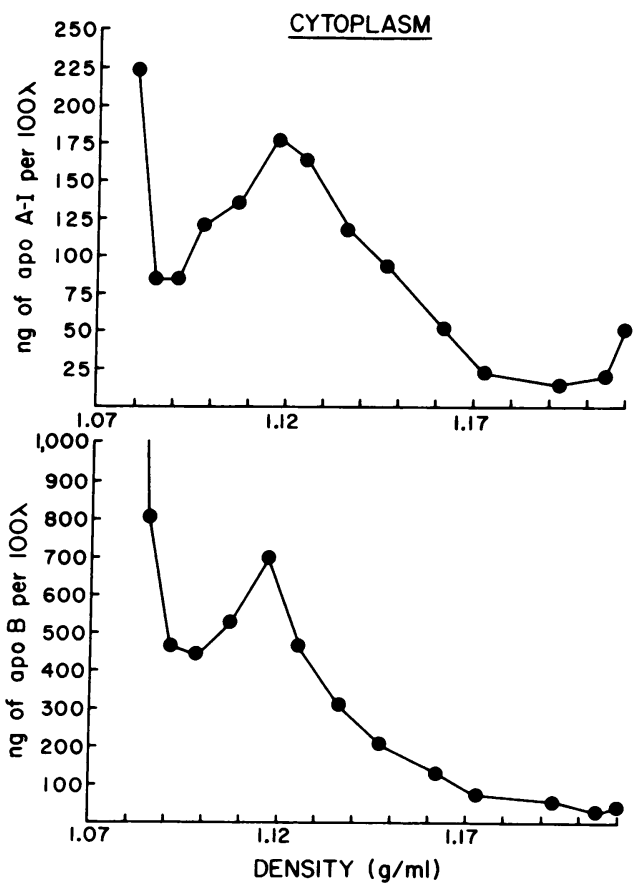

Figure 3. Apoprotein distribution of cytoplasmic HDL particles. The concentrated whole $\mathrm{HDL}$ fraction $(1.07-1.21 \mathrm{~g} / \mathrm{ml}$ ) was loaded on an isopyknic density gradient and measured by RIA. The peak at density $1.12 \mathrm{~g} / \mathrm{ml}$ for both apoproteins suggests an intracellular HDL population of particles.

The possibility that chylomicrons were being fragmented into HDL particles during the isolation procedure was also examined, by combining a cellular homogenate free of lipoproteins with lymph chylomicrons and then subjecting this solution to the preparative method, as described in Methods. Fig. 5 illustrates the apoprotein distribution across an isopyknic density gradient of the HDL fraction from this solution. In contrast to the previous density gradients, there is no peak of apoA-I or apoB in the middle of the gradient. Thus, the methodology to isolate intracellular lipoproteins does not produce an artifactual HDL particle from chylomicrons.

\section{Characterization of intracellular HDL}

Immunoelectrophoresis of HDL. To define mobility and apoprotein characteristics, immunoelectrophoresis was performed on the concentrated whole HDL fraction $(1.070-1.21 \mathrm{~g} / \mathrm{ml})$ of a cytoplasmic preparation (Fig. 6). In the alpha region of the gel, a precipitin arc against apoA-I is seen which extended into the pre-beta region, where an apoB precipitin arc is also seen. The $1.13-\mathrm{g} / \mathrm{ml}$ fraction from an isopyknic density gradient was then examined by immunoelectrophoresis (Fig. 7 B). This fraction, which contains only HDL particles (i.e., it is free of contamination by $d<1.070$ particles), also revealed a precipitin arc against apoA-I in the alpha region and against apoA-I and apoB in the pre-beta region. These findings suggested that there were at least two populations of intracellular HDL particles, one with apoA-I having alpha mobility and another with apoA-I and apoB having pre-beta mobility.

In an attempt to deplete the HDL fraction of the apoBcontaining particles, apoB antiserum was added to the HDL fraction to immunoprecipitate the apoB particles, and then 

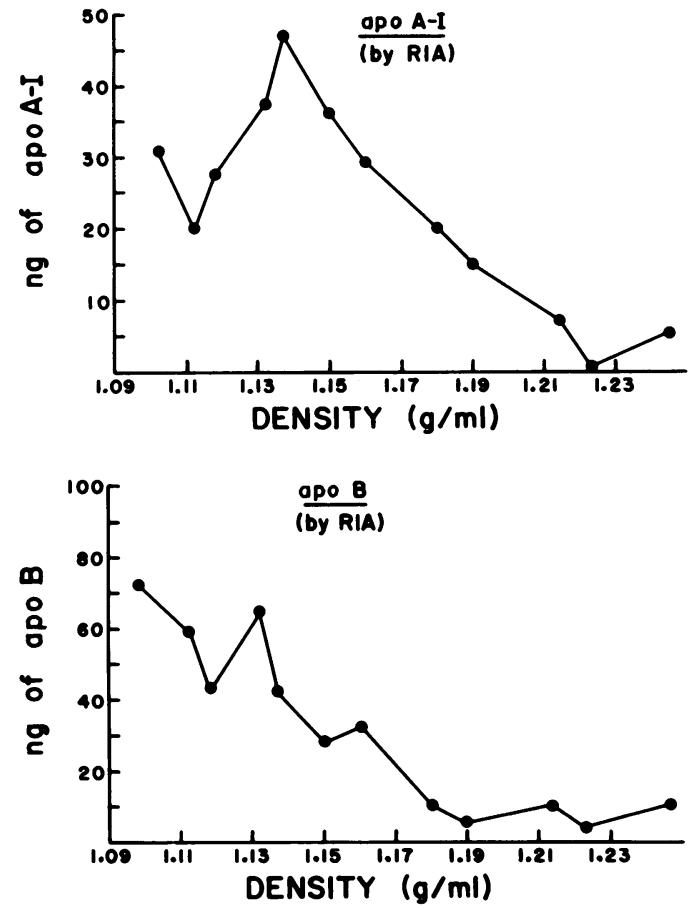

Figure 4. Apoprotein distribution of cytoplasmic HDL particles isolated from bile-ligated animals. The peak density for both apoproteins is $1.13 \mathrm{~g} / \mathrm{ml}$.

the supernatant was reexamined by immunoelectrophoresis (Fig. $7 \mathrm{C}$ ). The precipitin arc against apoA-I in the alpha region remained but the arcs against apoproteins A-I and B in the pre-beta region were not seen. This suggested that a particle containing apoB and apoA-I was removed, leaving an apoA-I particle relatively free of apoB with alpha mobility in the HDL fraction.

$\left[{ }^{3} \mathrm{H}\right]$ leucine incorporation studies. To determine whether the apoproteins found on intracellular HDL particles were newly synthesized, $\left[{ }^{3} \mathrm{H}\right]$ leucine was administered intraduodenally and a cytoplasmic preparation was made. The HDL fraction $(1.070-1.21 \mathrm{~g} / \mathrm{ml})$ was subjected to isopyknic density

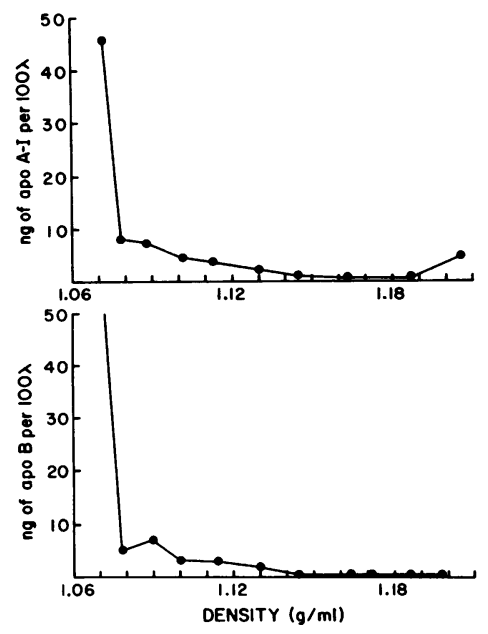

Figure 5. Apoprotein distribution of the $1.07-1.21 \mathrm{~g} /$ $\mathrm{ml}$ fraction from the lymph chylomicron-cellular homogenate preparation. The concentrated whole HDL fraction from the cytoplasmic method prepared from lymph chylomicrons and a lipoprotein-free cellular homogenate was loaded onto an isopyknic density gradient, and apoproteins were measured by RIA after a 72-h centrifugation.

The peak at the $1.07 \mathrm{~g} / \mathrm{ml}$ fraction are non-HDL particles. The lack of any peak of apoproteins in an HDL density range suggests that no HDL particles are isolated.



Figure 6. Immunoelectrophoresis of the concentrated whole HDL fraction from a cytoplasmic preparation. A precipitin arc is seen in the alpha and pre-beta region in the center lane against apoA-I, and another precipitin arc is seen in the pre-beta region against apoB. Rat plasma controls are in the outer lanes.

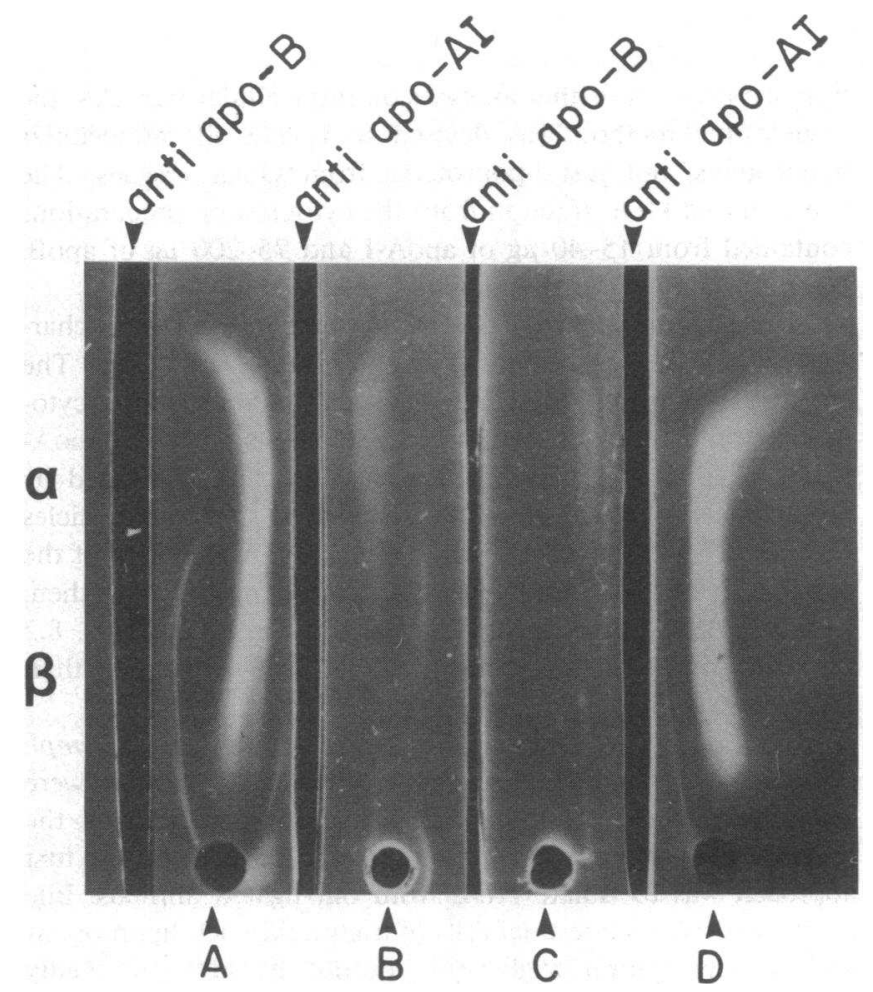

Figure 7. Immunoelectrophoresis of the $1.13-\mathrm{g} / \mathrm{ml}$ fraction from a cytoplasmic preparation (lane $B$ ) and of an apoB-depleted whole HDL fraction (lane $C$ ). In lane $B$, precipitin arcs are seen in the alpha region against apoA-I and in the pre-beta region against apoB. After immunoprecipitation of apoB particles, in lane $C$, a precipitin arc is seen only in the alpha region against apoA-I. Plasma controls are in lanes $A$ and $D$. 
gradient centrifugation. Fig. 8 shows the distribution of radioactivity across the density gradient. The pattern is identical to the mass distribution of apoA-I and apoB as measured by RIA, suggesting that apoproteins on the HDL particles being isolated are newly synthesized. The radiolabeled $1.13 \mathrm{~g} / \mathrm{ml}$ peak fraction was then delipidated and subjected to SDSpolyacrylamide gel electrophoresis, the gels were sliced, and radioactivity was determined. Three peaks of radioactivity were seen corresponding in retardation factor $\left(R_{f}\right)$ to apoB, apolipoprotein A-IV (apoA-IV), and apoA-I (Fig. $9 \mathrm{~A}$ ).

To confirm further that there were at least two particles in the HDL fraction, apoB antiserum was added to the 1.13-g/ $\mathrm{ml}$ fraction of the radiolabeled preparation, and apoB-containing apoproteins were immunoprecipitated with a second antibody. The supernatant and the precipitate were then delipidated and examined by SDS-gel electrophoresis (Fig. 9 B). The gel slices of the supernatant showed that although almost all of the radiolabeled apoB was removed by the immunoprecipitation, apoA-I and apoA-IV were left relatively undiminished. The precipitate concomitantly contained mostly apoB with minor amounts of radioactivity in apoA-I and apoA-IV (Fig. 9 C).

Lipid composition of HDL particles. The lipid composition of HDL particles from the Golgi of normal and bile-ligated animals and from a cytoplasmic preparation is seen in Table I, columns A-C. Column D shows the lipid composition of the HDL fraction post-depletion of apoB-containing particles. Apo-B particles were removed by double antibody immunoprecipitation with lipid-free antisera. The immunoprecipitate containing the apoB particle was not analyzed.

\section{Discussion}

This study describes the isolation of newly synthesized HDL particles from within intestinal epithelial cells, which is consistent with the hypothesis that the intestine is a site of origin of HDL. Previous studies have established that the intestine can synthesize chylomicrons and VLDL particles. These 250-3,000 $\AA$ particles are easily identified within the Golgi apparatus and



Figure 8. Distribution of radioactivity across a density gradient of an intracellular HDL fraction. $\left[{ }^{3} \mathrm{H}\right]$ leucine was administered into the duodenum, a cytoplasmic preparation was made, and the HDL fraction was isolated and subjected to isopyknic density gradient ultracentrifugation.

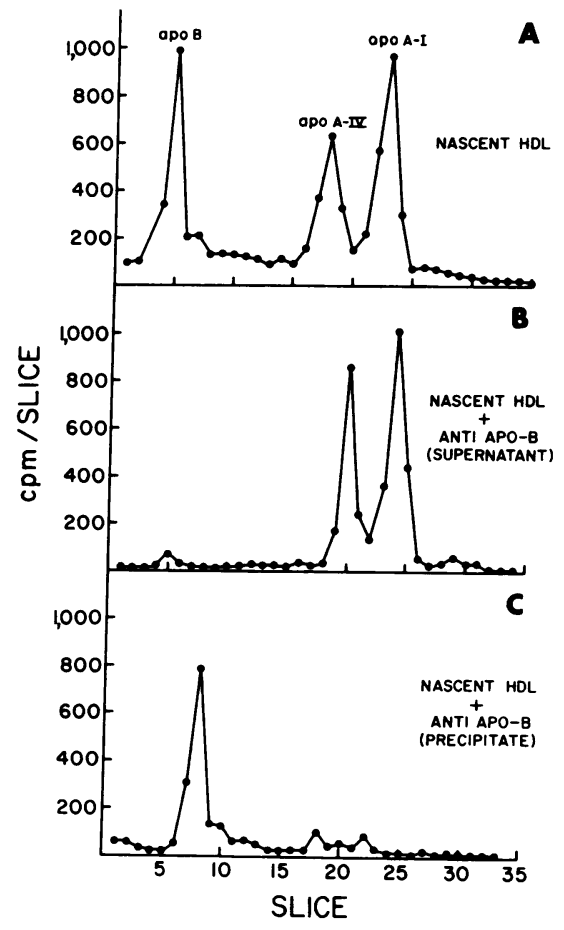

Figure 9. Radioactive incorporation of $\left[{ }^{3} \mathrm{H}\right]$ leucine into cytoplasmic intracellular HDL apoproteins. The $1.13-\mathrm{g} / \mathrm{ml}$ fraction from the density gradient of the radiolabeled HDL preparation was delipidated, the apoproteins were subjected to $5.6 \%$ SDS-polyacrylamide gel electrophoresis, and the radioactivity in each slice was determined. The $R_{f}$ of the peaks was $0.14,0.51$, and 0.66 , corresponding to apoB, apoA-IV, and apoA-I, respectively (27). (A) $1.13-\mathrm{g} / \mathrm{ml}$ fraction. $(B)$ Supernatant post-immunoprecipitation of the apoB particles by the use of a double antibody technique. $(C)$ Immunoprecipitate after addition of apoB antisera.

secretory vesicles of electron micrographs of rat and human epithelial cells (7). Intracellular forms of intestinal HDL have not been previously described. The idea that such particles might be synthesized in the intestine stems from studies of mesenteric lymph, which have shown that the lymph contains unique HDL particles (discoidal and small spherical) and that 75\% of newly synthesized intestinal apoA-I can be found in

Table I. Lipid Composition of Intestinal HDL

\begin{tabular}{lrrrr}
\hline & \multicolumn{2}{c}{ \% Composition } & & \\
\cline { 2 - 5 } & A & B & C & D \\
\hline Phospholipid & 48 & 41 & 40 & 38 \\
Cholesteryl ester & 24 & 22 & 12 & 17 \\
Triglyceride & 7 & 13 & 16 & 22 \\
Fatty acid & 18 & 20 & 23 & 13 \\
Cholesterol & 3 & 4 & 8 & 10 \\
& & & &
\end{tabular}

HDL particles were isolated and their lipid composition was determined by TLC. A, HDL fraction from a Golgi preparation (15 rats). B, HDL fraction from a Golgi preparation using bile-ligated rats (15 rats). C, HDL fraction from a cytoplasmic preparation $(n=3) . \mathrm{D}$, HDL fraction post-depletion of apoB particles from a cytoplasmic preparation $(n=3)$. 
the lymph HDL fraction during fasting (2-5). The size of an HDL particle (6-14 nm), however, precludes its accurate identification in an electron micrograph of an epithelial cell or a Golgi organelle. Thus, to prove that the intestine synthesizes HDL, it is necessary to isolate newly synthesized HDL particles from within the cell.

In this paper, we describe two different procedures to isolate HDL particles from epithelial cells. Preliminary experiments were performed by means of a modification of a procedure described by Weiser et al. to isolate a Golgi fraction from intestinal epithelial cells (9). A Golgi fraction with increased galactosyltransferase activity was successfully prepared. Though the appearance of the vesicles (as seen in Fig. $1 A$ ) suggests that there could be smooth microsomes in the fraction, the important point is that the fraction consists of organelles containing lipoproteins. We then attempted to rupture the organelles by several techniques, including sonication, passage through a 27-gauge needle, hypotonic shock, solubilization in $8 \mathrm{M}$ urea, and passage through a French pressure cell apparatus. The yields of HDL when these methods were used to rupture the Golgi fraction were low. During these investigations, Howell and Palade published a method that used $100 \mathrm{mM}$ sodium carbonate to resolve a hepatic Golgi fraction into membrane and content fractions and demonstrated the isolation of lipoproteins from the Golgi fraction $(10,11)$. We modified their isolation procedure for use with an intestinal preparation (see Methods) and found that by suspending the Golgi fraction in sodium carbonate, the yields of liberated lipoproteins were sufficient for electron microscopy, density gradient analysis, or lipid determination of an HDL fraction. The Golgi procedure in this paper is based on their method.

The HDL fraction isolated from Golgi vesicles contains 8.2-nm particles as seen by electron microscopy. The density of the particles, as determined by peak apoA-I and apoB concentration, is $1.11-1.14 \mathrm{~g} / \mathrm{ml}$. The lipid composition by TLC shows lecithin and cholesteryl ester as the major lipids. HDL isolated from the cytoplasmic preparation are $11.2-\mathrm{nm}$ particles with a density of $1.12 \mathrm{~g} / \mathrm{ml}$. Lipid composition shows lecithin, triglyceride, and cholesteryl ester as major lipids. To prove the cytoplasmic particles were synthesized in the intestine, $\left[{ }^{3} \mathrm{H}\right]$ leucine was administered intraduodenally and an $\mathrm{HDL}$ cytoplasmic fraction was isolated. The HDL fraction contained labeled apoA-I, apoB, and apoA-IV.

The cytoplasmic isolation method provides a 10-fold greater yield of lipoprotein than does the Golgi method. About 15\% of the cytoplasmic particles are the size of Golgi-derived particles, as determined by direct measurement of the diameter of the particles from the electron micrographs. Since the procedure isolates all lipoproteins from within the cell and since lipoproteins are probably undergoing a continuous modification from the time of assembly to actual secretion, it was not surprising that the density and size of HDL isolated from the cytoplasmic method were not exactly the same as those found in Golgi vesicles. Similarly, the density of HDL from bile-ligated animals differed slightly from that of HDL from normals, as might be possible with a major change in lipid flux across the enterocyte. The fact that both isolation methods yielded particles with high densities $(d>1.11 \mathrm{~g} / \mathrm{ml})$ and similar apoprotein and lipid characteristics warrants use of the cytoplasmic isolation method for lipoprotein analyses when increased yields are required.
The presence of apoB in an HDL fraction raised the possibility that there was more than one type of particle in the HDL fraction. Immunoelectrophoresis of the whole HDL fraction from a cytoplasmic preparation demonstrated that there was a precipitin arc against apoA-I in the alpha region and a precipitin arc against apoB and apoA-I in the pre-beta region. When apoB particles were depleted by immunoprecipitation and the immunoelectrophoresis was repeated, the alpha region still contained an arc against apoA-I, but no arcs were seen in the pre-beta region. Immunoprecipitation of the apoB particles from the radiolabeled HDL fraction also suggested that apoA-I (and apoA-IV) were not on the same particle as apoB. Thus, the cytoplasmic HDL fraction appeared to contain at least two particles with the same density but different apoprotein and mobility characteristics.

Since both the Golgi and cytoplasmic preparative procedures yielded an unexpected result, i.e., an apoB-containing particle in an HDL density range, it was important to prove that the particles in the HDL density range were not an artifact of the procedure, produced by fragmenting chylomicrons. Two experimental approaches were used to address this issue. It is known that bile diversion markedly depletes Golgi organelles and secretory vesicles of chylomicrons (19). Therefore, if HDL lipoproteins were being produced by the procedure from chylomicrons, then with bile ligation the characteristics of HDL should be significantly altered. This was not the case. Conversely, we attempted to produce HDL particles from chylomicrons. Lymph chylomicrons containing about twice the amount of apoA-I usually present in a starting cellular homogenate were added to a lipoprotein-free cytoplasmic homogenate and a cytoplasmic preparation was made from which lipoproteins were isolated. The $1.070-1.21 \mathrm{~g} / \mathrm{ml} \mathrm{HDL}$ fraction contained apoprotein and lipid, but the distribution across the density gradient did not reveal a population of HDL particles. In addition to these two experiments, the fact that the only newly labeled proteins on HDL particles corresponded in $\mathbf{R}_{\mathbf{f}}$ to known apolipoproteins suggests that the particles are not aggregations of lipid and intracellular protein produced during the isolation method. Finally, recombinant studies with apoprotein and lipid in vitro have shown that recombinant HDL lipoproteins do not form at temperatures $<20^{\circ} \mathrm{C}(20)$. Thus, although a cell fractionation study potentially may result in artifacts, we feel that this possibility has been reasonably excluded in the present study.

The apoA-I particle with alpha mobility isolated from the Golgi resembles a spherical HDL particle isolated from mesenteric lymph by Forester et al. This lymph 7.8-nm spherical particle, rich in apoA-I and phospholipid, has a density of $1.13-1.18 \mathrm{~g} / \mathrm{ml}$ and contains newly synthesized cholesteryl ester (4). It is possible, though unproven, that the small spherical particle in the lymph is the secreted form of the spherical apoA-I-rich intracellular HDL. The finding of the intracellular apoB-containing particle in the HDL fraction is unusual. The particle differs from the apoA-I particle in electrophoretic mobility and apoproteins, though size, density, and lipid composition are similar. Theoretically, a particle with density of $1.12 \mathrm{~g} / \mathrm{ml}$ and size of $8-10 \mathrm{~nm}$ has a calculated molecular weight of $352,000-475,000$, which is compatible with the molecular weight of intestinal apoB $(248,000)(21)$. Furthermore, an apoB molecule occupies a volume of 295 $\mathrm{nm}^{3}$, which would easily fit on a spherical particle $10 \mathrm{~nm}$ in 
diameter, whose calculated volume is $523 \mathrm{~nm}^{3}$. It is interesting that the finding of a nascent form of $\mathrm{HDL}$ that contains apoB is not without precedent. Howell and Palade found apoB in the HDL fraction of hepatic Golgi lipoproteins (11) and Fainaru et al. isolated a spherical HDL particle from a rat hepatic perfusate with beta mobility and with apoB as the major apoprotein (22). The apoB intracellular particle may be an immature chylomicron or a form of intestinally derived lymph LDL or HDL.

Our findings raise several questions which require further investigation. We found no discoidal forms of $\mathrm{HDL}$ in the cell such as were found in the lymph (4). Neither did Howell and Palade find discoidal HDL in the hepatic Golgi fraction such as were found by Hamilton et al. in hepatic perfusates (23). Our studies were not done in the presence of dithiobis(nitrobenzoic acid), a lecithin/cholesterol acyl transferase (LCAT) inhibitor, as LCAT is not found in the intestinal cell (24). The intestinal lymph and hepatic perfusate studies were done with an LCAT inhibitor. Whether the addition of dithiobis(nitrobenzoic acid) will affect the isolation of intestinal intracellular lipoproteins and whether a discoidal form exists in the cell are not known.

The molecular or isoelectric forms of apoA-I and apoA-IV on the intracellular particle were not determined. Gordon et al. have shown that apoA-I is synthesized as a preproprotein from which the signal segment is cotranslationally cleaved, forming pro-apoA-I (25), which is similar to the basic isoform of apoA-I found by Zannis et al., as secreted by human intestinal organ culture (26). The site of proteolytic conversion of the proprotein to the more acidic plasma form of apoA-I is not known but is thought to be extracellular. Identification of the form of apoA-I on a nascent intestinal HDL particle isolated from the Golgi apparatus and a cytoplasmic fraction might add to an understanding of apoprotein processing. ApoB found on the HDL particle is probably the low molecular weight form thought to be synthesized by the intestine since the $R_{f}(0.14)$ is compatible with the low molecular weight form, as isolated on $5.6 \%$ acrylamide gels (27).

The isolation of intracellular intestinal particles in an HDL density range raises important issues about lipoprotein assembly. As was found in the liver, the intestine seems to be able to synthesize a broad range of lipoproteins, from a $6.0-\mathrm{nm} \mathrm{HDL}$ particle to a 300 -nm chylomicron. As currently hypothesized, lipoproteins are assembled between the rough endoplasmic reticulum and the Golgi apparatus, modified in the Golgi, and then packaged into secretory vesicles. The factors determining chylomicron or HDL formation are not understood. It is possible that all lipoproteins begin from a pool of small, dense particles to which, with appropriate stimulation, triglyceride and cholesterol are added. Alternatively, there may be a separate pathway of assembly for a chylomicron that is activated by fat absorption and another pathway for HDL formation, as is suggested by the continued presence of an apoB particle after biliary diversion and consequent diminution of triglyceride flux. The modifications intracellular lipoproteins undergo after initial assembly are also not understood. There might be a constant interaction and remodeling of the surface and core components among the various lipoproteins within the cell, as occurs upon secretion. The methodology developed to isolate intracellular HDL should facilitate continued investigation of the spectrum of intracellular lipoproteins and the factors that influence lipoprotein synthesis and secretion.

\section{Acknowledgments}

We thank Drs. Alan Tall, Nicholas Davidson, Charles Bisgaier, and Amy Samuelson for helpful advice and Ms. Deborah Russell for technical assistance. The apoB RIA was developed by Dr. Samuelson. The electron microscopy of the Golgi vesicles was performed by Jesse Kirshman.

This work was supported by National Institutes of Health grants AM 21367, HL 21006, and AM 07330.

\section{References}

1. Windmueller, M. G., and A.-L. Wu: 1981. Biosynthesis of plasma apolipoproteins by rat small intestine without dietary or biliary fat. J. Biol. Chem. 256:3012-3016.

2. Glickman, R. M., and P. H. R. Green. 1977. The intestine as a source of apolipoprotein A-I. Proc. Natl. Acad. Sci. USA. 74:25692573.

3. Bearnot, H. R., R. M. Glickman, L. Weinberg, P. H. R. Green, and A. R. Tall. 1982. Effect of biliary diversion on rat mesenteric lymph apolipoprotein A-I and high density lipoprotein. J. Clin. Invest. 69:210-217.

4. Forester, G. P., A. R. Tall, C. L. Bisgaier, and R. M. Glickman. 1983. Rat intestine secretes spherical high density lipoproteins. J. Biol. Chem. 258:5938-5943.

5. Green, P. H. R., A. R. Tall, and R. M. Glickman. 1978. Rat intestine secretes discoid high density lipoprotein. J. Clin. Invest. 61:528-534.

6. Mahley, R. W., B. D. Bennett, D. J. Morre, M. E. Gray, W. Thistlethwaite, and V. S. LeQuire. 1971. Lipoproteins associated with the Golgi apparatus isolated from epithelial cells of rat small intestine. Lab. Invest. 25:435-444.

7. Swift, L. L., P. D. Soule, M. E. Gray, and V. S. LeQuire. 1984. Intestinal lipoprotein synthesis. Comparison of nascent Golgi lipoproteins from chow fed and hypercholesterolemic rats. J. Lipid Res. 25:113.

8. Sabesin, S. M., and S. Frase. 1977. Electron microscopic studies of the assembly, intracellular transport, and secretion of chylomicrons by rat intestine. J. Lipid Res. 18:496-511.

9. Weiser, M., M. M. Newmeier, A. Quaroni, and K. Kirsch. 1978. Synthesis of plasmalemmal glycoproteins in intestinal epithelial cells. J. Cell Biol. 77:722-734.

10. Howell, K. E., and G. E. Palade. 1982. Hepatic Golgi fractions resolved into membrane and content subfractions. J. Cell Biol. 92:822832.

11. Howell, K. E., and G. E. Palade. 1982. Heterogeneity of lipoprotein particles in hepatic Golgi fractions. J. Cell Biol. 92:833845.

12. Tall, A. R., C. B. Blum, G. P. Forester, and C. A. Nelson. 1982. Changes in the distribution and composition of plasma HDL after ingestion of fat. J. Biol. Chem. 257:198-207.

13. Glickman, R. M., and K. Kirsch. 1973. Lymph chylomicron formation during the inhibition of protein synthesis. Studies of chylomicron apoproteins. J. Clin. Invest. 52:2910-2920.

14. Glickman, R. M., A. Kilgore, and J. Khorana. 1978. Chylomicron apoprotein localization within rat intestinal epithelium: studies of normal and impaired lipid absorption. J. Lipid Res. 19:260-268.

15. Fairbanks, G., T. L. Steck, and D. F. H. Wallach. 1971. Electrophoretic analysis of major polypeptides of the human erythrocyte membrane. Biochemistry. 10:2606-2617.

16. Lowry, O. M., N. J. Rosebrough, A. K. Farr, and R. J. Randall. 
1951. Protein measurement with the Folin phenol reagent. J. Biol. Chem. 193:265-275.

17. Folch, J., M. Lees, and G. H. Sloane Stanley. 1951. A simple method for the isolation and purification of total lipids from animal tissues. J. Biol. Chem. 226:497-509.

18. Katz, S. S., G. G. Shipley, and D. M. Small. 1976. Physical chemistry of the lipids of human atherosclerotic lesions. J. Clin. Invest. 58:200-211.

19. Jones, H. L., and R. K. Ockner. 1971. An electron microscopic study of endogenous VLDL production in the intestine of rat and man. J. Lipid Res. 12:580-589.

20. Hirz, R., and Scanu, A. M. 1970 . Reassembly in vitro of a serum high-density lipoprotein. Biochim. Biophys. Acta. 207:364-367.

21. Krishnaiah, K. V., L. F. Walker, J. Borensztajn, G. Shonfeld, and G. S. Getz. 1980. Apolipoprotein B variant derived from rat intestine. Proc. Natl. Acad. Sci. USA. 77:3806-3810.

22. Fainaru, M., T. E. Felker, R. L. Hamilton, and R. J. Havel. 1977. Evidence that a separate particle containing B-apoprotein is present in HDL from perfused rat liver. Metab. Clin. Exp. 26:9991004.

23. Hamilton, R. L., M. C. Williams, C. J. Fielding, and R. J. Havel. 1976. Discoidal bilayer structure of nascent HDL from perfused rat liver. J. Clin. Invest. 58:667-680.

24. Haugen, R., and K. R. Norum. 1976. Coenzyme A-dependent esterification of cholesterol in rat intestinal mucosa. Scand. J. Gastroenterol. 11:615-621.

25. Gordon, J. I., H. F. Sims, S. R. Lentz, C. Edelstein, A. M. Scanu, and A. W. Strauss. 1983. Proteolytic processing of human preproapolipoprotein A-I. J. Biol. Chem. 258:4037-4044.

26. Zannis, V. I., D. M. Kurnit, and J. L. Breslow. 1982. Hepatic apoA-I and apoE and intestinal apoA-I are synthesized in precursor isoprotein forms by organ cultures of human fetal tissues. J. Biol. Chem. 257:536-544.

27. Tall, A. R., P. H. R. Green, R. M. Glickman, and J. W. Riley. 1979. Metabolic fate of chylomicron phospholipids and apoproteins in the rat. J. Clin. Invest. 64:977-989. 